\title{
ON THE DEVELOPMENT OF SYSTEMS OF MEN AND MACHINES
}

H. D. Mills, IBM Corporation and The Johns Hopkins University

With Appendix by R. C. Linger, IBM Corporation

\section{ABSTRACT}

We formulate the development of systems of men and machines as a programing problem for multiprocessing in which some processors are men and some are machines. In this way, users guides, training courses, etc., are determined from processing requirements, just as machine specifications, which are consistent with the objectives of the total operation. An Appendix illustrates this idea in miniature for a supermarket checkout operation.

\section{Systems of Men and Machines}

our topic is the architecture, implementation, and operation of large systems of men and machlues in some definite and coherent enterprise - managing a business, operating an airline reservation system, ruming a goverment agency, getting men to the moon and back, etc. In such systems there are many kinds of men (and women) -- managers, clerks, spectalists of various kinds, and machtne tenders; there are also many kinds of machines -- computers, terminals, sensors, actuators, communications equipment, etc.

Ordinarily computer programming is regarded as part of the machine side of the system, and programmers as part of the machine tenders. We bring a different view -- that the architecture of the operations of the enterprise is programming, as well. Our thesis is that modern principles of programming -- forced on us out of necessity in dealing with machines of much logical capability but no common sense -- can play as vital a role in bringing systematic discipline and standards into all phases of large systems development.

For this point of view we escalate the concept of programming to that of providing comprehensive instructions for either man or machine activitles. The operations of an enterprise becomes a multiprocesssing operation of men 
and machines; then the architecture of the operations defines the configurations and types of men and machines in the system, and the programs which direct each type of man and machine. For example, a users guide becomes one part of a cooperating system of programs operating in separate processors (the user and a machine).

of course, the characteristics of man and machine are quite different in such a system, just as machines are different among themselves. And yet their architectural properties can be treated in a uniform way. For example, in deciding on a particular machine requirement, various considerations of physical or logical capability will arise, as well as whether the requirements can be met with off-the-shelf equipment; these same considerations apply to a man requirement, in common terms -- e.g., how many letters can a postal clerk sort in an hour, and can this be done with minfmal training, etc.

It is well understood that men and machines do well at quite different things. Machines are good at doing what they are told to do, very rapidiy and accurately. Men are good at using common sense -- even disobeying instructions when they are obviously misconcelved; at pattern recognition -- discovering information by no spectal or dependable process; and at invention -- creating a new idea for the enterprise to act on. One simple, but very fimportant form of pattern recognition is the translation of human speech to and from machine readable text. This is routine for clerical activities that interface with persons outside the enterprise -- e.g., in an airline reservation system.

It may be asked how the concept of programing applies to men, which operate so differently and unpredictably, compared to machines. It applies by noting that programs are used in a local way by machines -- i,e., at this moment, under these conditions, do this next. A good deal of programming on the man side is already subsumed under general instructions and common sense - - if the telephone rings, answer 1t; if you want to execute a program, keypunch a job deck and submit it to the operator. We have no intention of explicitly programing human activities now done by general instructions and cormon sense; the typical level of human programing envisioned is that which is found in users guides, operating instructions, etc., associated with machine operations. 
Principles of Programming for Men and Machines

The recent, twenty-five year, history of computer programming has seen an explosive and traumatic growth of human activity and frustration in attempting to realize the promise and potential of electronic and electromechanfcal devices for processing data in science, industry and government. Out of this history has come the stored program computer; programming languages, compllers, and librarles; and new technical foundations for programing computers, e.g., as propounded by McCarthy [ 4], Dijkstra [ I], Hoare [ 3], and Wirth [10]. In this period the computer has been the center of attention. In the beginning, the numerical computing power was so great, compared to manual methods, and the availability so 1 imited, that men readily adapted to this new tool - from decimal to binary, from equations to algorithms. But in a short time, the remarkable possibilitles for more general data processing (nonnumertcal) were realized, and a new industry was born in just a few years. In the later part of this period (up to now) the large data processing systems appeared -- management information systems, afrline reservation systems, space tracking systems, etc. Even then, although human factors were considered, these systems were conceived primarily as data processing systems, which responded to users. But in our proposed perspective, the users are as much a part of the multiprocessing enterprise as the machines and their programs.

Thus, whereas the computer has forced us to find more effective programing principles than we would otherwise have, it has also warped our sense of perspective. By this time, simple human factors questions are in better focus. There is enough data processing power available to tnvest part of it in creating more human-like interfaces than binary and machine code. But in extending programming to instructing men, with their entirely different characteristics, additional fdeas and principles are needed, such as

1. Languages. The programming languages used in the architecture of systems of men and machines need be near natural languages. The difficulties of processing natural languages are well known and that is not proposed. Rather, what is proposed is a "naturalization" of processable programing languages which is close enough for use as a dialect of natural language by nonprogrammers of the enterprise. Sammet [ 8] and Zemanek [11] discuss both sides of this. 
2. Procedures. The concept of procedure should be extended to include indefinite procedures where it is not possible or desirable to define them. In simple cerms, "find values for these variables so that these equations hold" (Wilkes discusses this in [9]), or more complex, "make sure no one"s feelings are hurt".

3. Interactions. The principal subject of the architecture is mult1processing - the conduct of the operation of the eaterprise through programs distributed to men and machines. The creation of such programs in an orderly, systematic way will require a new and fundamental development beyond programming principles for synchronous operations. The 1dea of the Petri net [ 7] may be an embryonic step in such a development. Dennis iliustrates Petri nets as multiprocessing control mechanisms, In [2].

\section{Architecture Principles}

A system depends on its components. The architecture of a system specifles the types of men and machines required, as well as how they are to interact as a system opexation, $i . e$. , the selection and arrangement of the system components, as we11 as detailed instructions for their behavior. In the case of machines, the usual considerations apply - define feasible requirements, elther alxeady embodied in existing machines, or possible with special development where justified; tradeoffs and comparisons with alternative approaches, even with manual approaches where feasible, etc. In the case of men, the systen architect is frequently shortsighted. There is a reason; it is much more difficult to predict a human performance than a machine performance. Sometimes the human falls to live up to a requirement. But often the human will exceed a requirement in a totally unexpected way, by acquiring a skill not imagined possible beforehand. This is happening with computer programmers right today, who are beginning to program with a precision not believed possible five years ago. It happened with typing when touch typing was introduced early in this century.

In recrospect, it is easy to identify a pitfall in overestimating machine possibilities, leading to a common scenario in many large systems in recent history, which put a "man in the $100 \mathrm{p}$ " at the last moment, with marginal operational results. In such a case, the operation was originally planned as completely automatic, depending on some key algorithm coften involving some form of pattern recognition); as a result of the planning, the data processing functions surrounding the algorithm were developed in parallel 
according to a general system design; then at the last moment, the performance of the key algorithm proves inadequate, and the man is brought into the loop, with two costs:

1. The human factors are bad (e.g., interacting with programs which require long, fixed argument 1ists); these can be fixed up.

2. There is a lost opportunity in not having a better trained man in the loop. The effort on algorithm development is frequently different than that required for insight development for a human executing an indefintte procedure, and the time is lost, anyway.

These operational experiences lead to the following principles of systems architecture:

1. Components. Regard men and machines as equal status components for system operations with equal requirements for development, state-of-art projections, and improvement, according to their own characteristics.

2. Evolution. Plan on the unexpected, by well-structured interfaces, that permit the replacement of components by improved versions which perform identical functions more effectively, Parnas [ 6] deals with this interchangability by axiomatizing such interfaces.

3. Integrity. Value system integrity above all else, by requiring that the multiprocessing operation of men and machines be described and scrutinized according to the best principles of programming -- particularly with respect to methods of specification and validation of programs. Note especially the technique defined by Wirth [10] and Mills [ 5].

In an Appendix dealing with a minature problem, R. C. Linger illustrates the idea of programming an operation through a set of abstract processes which only later are specialized to either men or machines, depending on their requirements. It is an easy transition from man as a user to man as a processor and yet it seems a critical one in providing system coherence and integrity with respect to a given operation. 
Literatur

[1] 0. - . Dahl, E. W. Dijkstra and C. A. R. Hoare, Structured Programming, Acadenic Press, London. 1972.

12] J. B. Dennis, "Concurrency in Software Systems", Lecture Notes in Economics and Mathematical Systems, 81 Advanced Course on Software Engineering (Ed. F. L. Bauex), Springer-Verlag, Berlin, Heidelberg, New York. 1973.

[3] C.A.R. Hoare, "An axlomatic basis for computer programming", CACM 12. 1969. pp. 576-580, 583.

[4] J. McCarthy, "A basis for a mathematical theory of computation", Computer Programing and Formal Systems, (Eds. P. Braffort and D. Hirschberg), North-Holland Publishing Company. 1963. pp. 33-70.

[5] H, D. Mil1s, "The new math of computer programing", CACM 17, 1975. (To appear).

[6] D. L. Parnas, "A technique for software module specification with examples", CACM 15, 5. May 1972. pp. 330-336

[7] C. A. Petri, Communications with Automata. Supplement 1 to Technica1 Report RADC-TR-65-377, Vo1. 1, Griffiss Air Force Base, New York. 1966. (Originally published in German: Kommunikation mit Automaten, University of Bonn, 1962).

[8] J. E. Sammet, "The use of English as a programing language", CACM 9, 3. March 1966. pp. 228-230.

[9] M. Wilkes, "Constraint-type statements in programming languages", CACM 7. 1964. pp. 587-588.

[10] N. Wirth, Systematic Programing: An Introduction, PrenticeHa11, Englewood Cliffs, New Jersey. 1973.

[11] H. Zenanek, "Semiotics and programming languages", CACM 9, 3. March 1966. pp. 139-143. 
Appendix

SUPER MARKET CHECKOUT AS A MAN-MACHINE MULTIPROCESSING ACTIVITY

R. C. Linger, IBM Corporation

Consider the problem of spectfying a design for a super market checkout operation. Several possibilities come to mind; a man with an adding machine and cashbox, a man with a cash register, a man with an OCR device which reads encoded prices, etc. Whatever the final configuration, we can begin our design with a procedural description of the checkout process itself. In this view, the dynamics of the process are of central interest. That is, we do not begin with a static description of components which must somehow fit together in a presumed system, but rather begin with a process which can be shown to work, and derive required (and possibly alternate) component configurations of men and machines from it. Our tentative first refinement is:

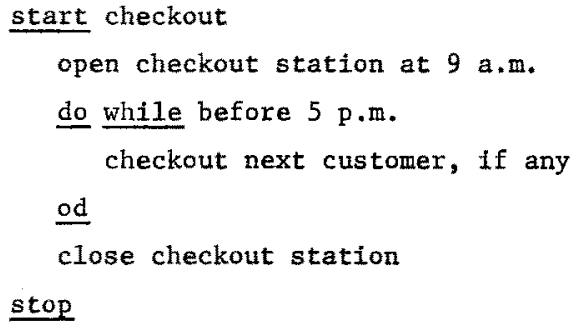


Here the functions of man and machine begin to emerge; presumably they will cooperate to "total cost of all items" through manual keystroke entry or OCR input, and the man wil "bag all items," without mechantcal assistance. We Identify "before 5 p.m." as a man predicate, In view of the human propensity for clockwatching, which hopefully yields to common sense to complete checkout of customers watting in line at quitting time! The possibility of "exception processing" arises in this procedure, as when the machines break down, or a coffee break is desired. These situations are analogous to the interrupt handing facilites of modern computers.

An aspect of the activity distribution between man and machine can be explored through elaboration of the process to "total cost of al1 items:"

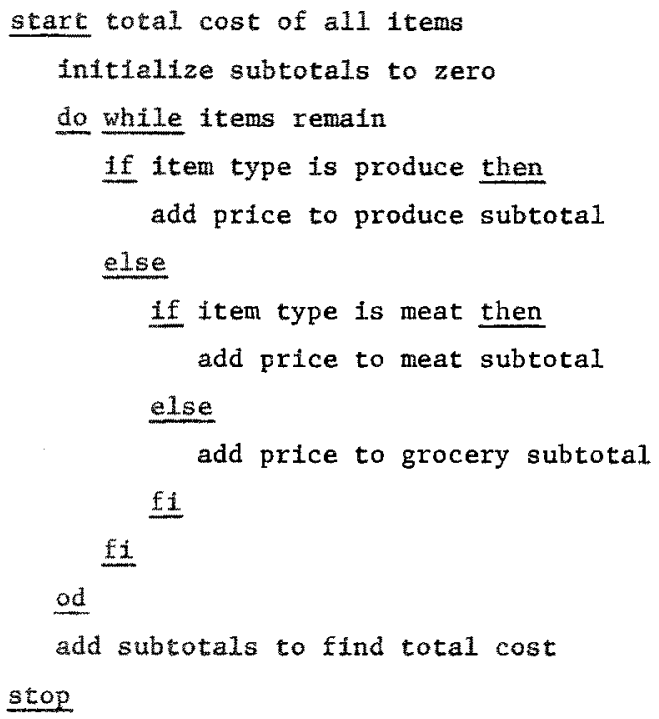

The cooperating give and take actions of man and machine appear as an abstract process here: "items remain" is likely a man predicate, while the "item type" expressions appear to be machine predicates set by man. The subtotal accumulations are best handled as machine functions. 
We can establish concrete procedures for man and machine by defining an interface between them. In 11lustration, consider an electromechanical cash register with control keys as follows:

$\begin{array}{ll}0,1, \ldots, 9 \\ \text { I } & \\ \text { P } & \\ \text { M } & \\ \text { G } & \\ \text { T } & \end{array}$

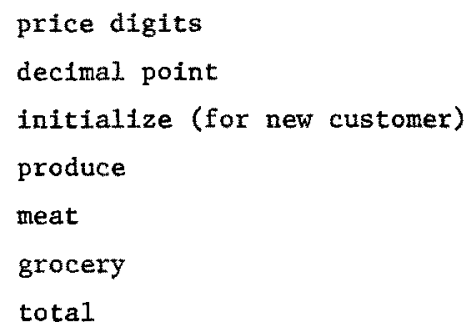

For this interface, the man procedure becomes

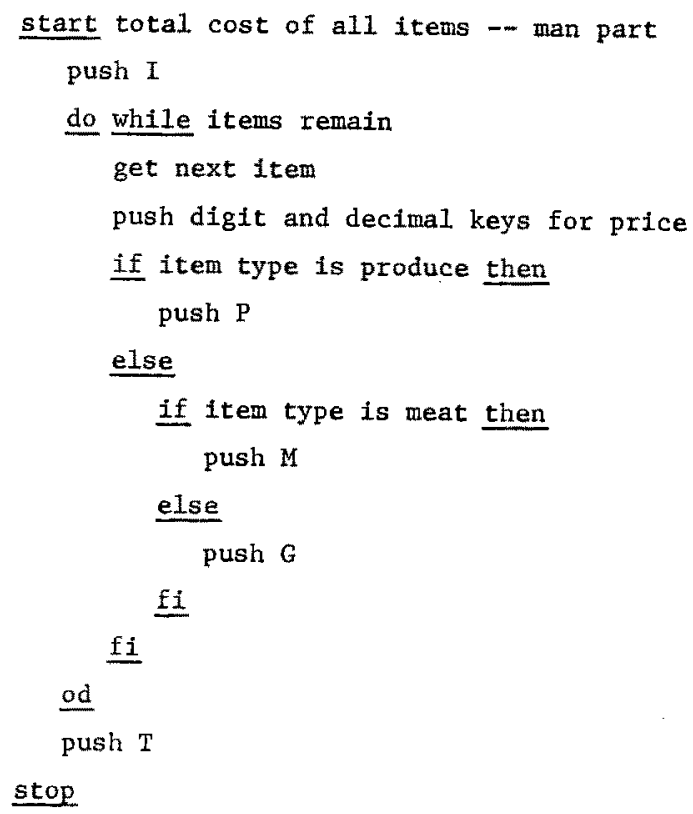

and the corresponding machine procedure is: 


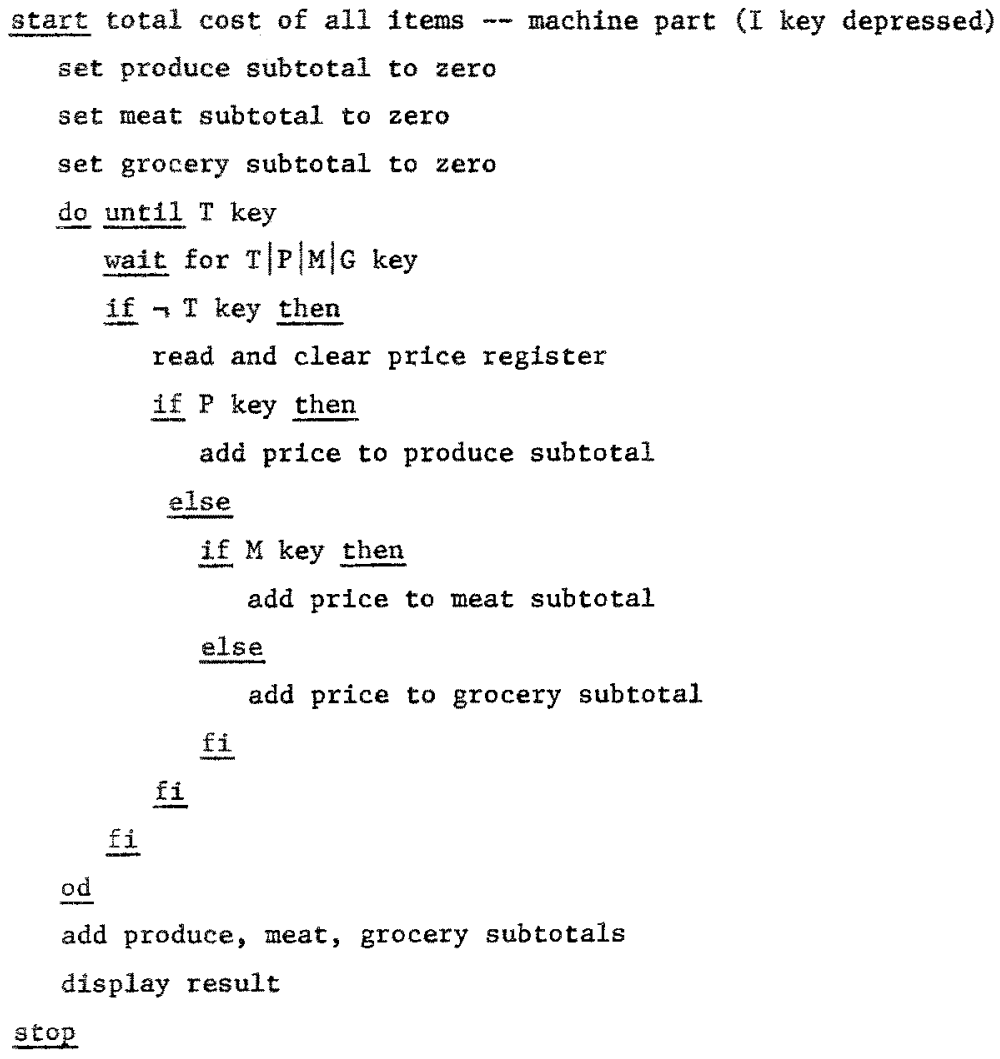

A different set of procedures derive from an alternate interface, as with a cash register using $O C R$ input, and equipped with only $I$ and $T$ keys. In this case the man procedure simplifies to

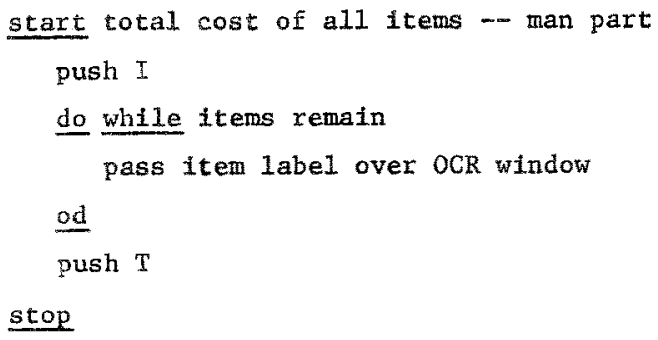

